\title{
Centralizer of an Ergodic Measure Preserving Transformation
}

\author{
By \\ Motosige OsIKAWA*
}

\section{$\S 1$. Tntroduction}

Let $T$ be an ergodic measure preserving transformation of a Lebesgue measure space $(\Omega, \mathfrak{B}, P), P(\Omega)=1$, that is, $T$ is a one to one mapping from $\Omega$ onto itself, bimeasurable $(T \mathfrak{B}=\mathfrak{B})$, measure preserving $\left(P\left(T^{-1} A\right)=P(A)\right.$ for $A$ in $\mathfrak{B}$ ) and ergodic (every measurable function $f(\omega)$ with $f(T \omega)=f(\omega)$ a.e. is constant a.e.). For measure preserving transformations $U$ and $U^{\prime}$ we write $U=U^{\prime}$ if $P\left(U \omega \neq U^{\prime} \omega\right)=0$ and $U \neq \leq U^{\prime}$ otherwise. A measure preserving transformation $U$ of $(\Omega, \mathscr{B}, P)$ is called a $p$-th root of $T(p \geqq 2)$ if $U^{p}=T$. A 1-parameter group $\left\{U_{t}\right\}$ of measure preserving transformations of $(\Omega, \mathfrak{B}, P)$ (i.e. $U_{t+s}=U_{t} U_{s}$ for $\left.-\infty<t, s<+\infty\right)$ is called a measurable flow if $(\omega, t) \rightarrow U_{t} \omega$ is a measurable mapping from $\Omega \times \mathbb{R}$ onto $\Omega$. If there exists a measurable flow $\left\{U_{t}\right\}$ with $U_{1}=T, T$ is said to be embeddable in a measurable flow. The existence of a $p$-th root or an embedding measurable flow has been one of problems in ergodic theory.

It is obvious that the existence of an embedding measurable flow of $T$ implies the existence of a $p$-th root of $T$ for every $p \geqq 2$ and also that a $p$-th root of $T$ (if exists) and an embedding measurable flow of $T$ (if exists) are ergodic.

A measure preserving transformation $U$ of $(\Omega, \mathfrak{B}, P)$ is said to commute with $T$ if $U T=T U$. We denote by $C(T)$ the group consisting of all measure preserving transformations each of which commutes with $T$ and call it the centralizer of $T$. Since a $p$-th root of $T$ (if exists) and a transformation $U_{t}$ for fixed $t$ in an embedding measurable flow $\left\{U_{t}\right\}$ of $T$ (if exists) are in $C(T)$, we may expect to solve the existence problem of roots and an embedding measurable

Communicated by H. Araki, September 8, 1980.

* Department of Mathematics, College of General Education, Kyushu University, Fukuoka 810, Japan. 
flow of some kind of ergodic transformation by determining its centralizer. A transformation $U$ in $C(T)$ is called to commute irrationally with $T$ if $T^{n} \neq U^{m}$ except for $n=m=0$. Whether an ergodic transformation commuting irrationally with given ergodic transformation exists or does not is a new problem.

Blum and Friedman [1], [2] discussed the existence problem of roots for ergodic transformations with discrete spectrum and constructed, for instance, an example of such transformation without any roots. Chacon [3], [4] gave an example of an ergodic transformation with continuous spectrum having no square root and one having no roots. Ornstein [10] gave an example of ergodic transformation $T$ with $C(T)=\left\{T^{n}: n \in \mathbb{Z}\right\}$ which, in consequence, has no roots nor an ergodic transformation commuting irrationally with it. In [11] he showed that every Bernoulli shift is embeddable in a measurable flow.

In Section 2 using the property of an invariant $\chi_{U}$ of a transformation $U$ in $C(T)$ which was introduced by $T$. Hamachi [6] we study the existence problem of a $p$-th root and an embedding measurable flow for ergodic transformations with pure point spectra. We show also that for an ergodic transformation with pure point spectrum there exists an ergodic transformation which commutes irrationally with it. In Section 3 we determine the $C(T)$ for a kind of transformations and give the following examples of ergodic transformations: (1) one not having any root, (2) one having only square root but no others, (3) one having a $2^{n}$-th root for any positive integer $n$ but no others, (4) one having a $n$-th root for any positive integer $n$ but not embeddable in a measurable flow, and (5) one whose commutant is not commutative. In Section 4 we give two examples of normalizers of ergodic non-singular transformations; one of them is one not having a square root and the other is one having any root but not embeddable in a measurable flow.

The author would like to thank I. Miyawaki for his contribution to the proof of Theorem $6(2)$.

\section{§2. Invariant $\chi_{U}$ and Transformation with Pure Point Spectra}

Let $T$ be an ergodic measure preserving transformation of a Lebesgue measure space $(\Omega, \mathfrak{B}, P), P(\Omega)=1$. A number $\gamma$ in the unit interval $[0,1)$ and a function $f_{\gamma}(\omega)$ are called a point spectrum and an eigenfunction, respectively, of $T$ if $f_{\gamma}(T \omega)=\exp (2 \pi i \gamma) f_{\gamma}(\omega)$ for a.e. $\omega$ in $\Omega$ and $\left|f_{\gamma}(\omega)\right|=1$. We denote by $S_{p}(T)$ the set of all point spectra of $T$. It is a countable subgroup of $[0,1)$ 
with respect to the additive operation modulo 1.

Lemma $\mathbb{1}([6])$. Let $T$ be an ergodic measure preserving transformation of a Lebesgue measure space $(\Omega, \mathfrak{B}, P), P(\Omega)=1$, then for a transformation $U$ in $C(T)$ there is a homomorphism $\chi_{U}$ from $S_{p}(T)$ into $[0,1)$ such that $f_{\gamma}(U \omega)$ $=\exp \left(2 \pi i \chi_{\nu}(\gamma)\right) f_{\gamma}(\omega)$ for a.e. $\omega$, for $\gamma$ in $S_{p}(T)$ and an eigenfunction $f_{\gamma}(\omega)$.

Proof. Since $f_{\gamma}(U \omega) / f_{\gamma}(\omega)$ is a $T$-invariant function and $T$ is ergodic, there is a constant $\chi_{U}(\gamma)$ such that $f_{\gamma}(U \omega) / f_{\gamma}(\omega)=\exp \left(2 \pi i \chi_{U}(\gamma)\right)$ for a.e. $\omega$. This constant $\chi_{U}(\gamma)$ does not depend on the choice of an eigenfunction $f_{\gamma}(\omega)$ for $\gamma$ and $\chi_{U}$ is a homomorphism from $S_{p}(T)$ into $[0,1)$.

Corollary 2. If $S_{p}(T)=\{0\}$ then for any ergodic transformation $U$ in $C(T) \quad S_{p}(U)=\{0\}$.

Proof. Obvious from Lemma 1.

Lemma 3. (1) For $U$ and $V$ in $C(T)$

$$
\chi_{U V}(\gamma)=\chi_{U}(\gamma)+\chi_{V}(\gamma)(\bmod 1) \text { for } \gamma \text { in } S_{p}(T) \text {. }
$$

Especially, $\chi_{U^{n}}(\gamma)=n \chi_{U}(\gamma)(\bmod 1)$ for $\gamma$ in $S_{p}(T)$ and $n$ in $Z$.

(2) $\chi_{T}(\gamma)=\gamma$ for $\gamma$ in $S_{p}(T)$.

(3) If $U$ in $C(T)$ is ergodic, $\chi_{U}(\gamma)=0$ implies $\gamma=0$.

Proof. Obvious.

Theorem ([2]). If $1 / n$ is in $S_{p}(T)$ for some integer $n$ with $(n, p) \neq 1$ then there is no p-th root of $T$, where $(n, p)$ is the greatest common measure of $n$ and p.

Proof. Assume that there is a $p$-th root $U$ of $T$. Since $p \chi_{U}(\gamma)=\gamma(\bmod 1)$ for $\gamma$ in $S_{p}(T)$, there is an integer $q$ such that $\chi_{U}(1 / n)=(1+n q) / n p$. Since $n \chi_{U}(1 / n)=0,(1+n q) / p$ is an integer, contradiction to $(n, p) \neq 1$.

An ergodic measure preserving transformation $T$ of a Lebesgue measure space $(\Omega, \mathfrak{B}, P), P(\Omega)=1$ is said to have pure point spectra if there is a complete orthonormal system of $L^{2}(\Omega, P)$ consisting of eigenfunctions of $T$.

Lemma 5. Let $T$ be an ergodic measure preserving transformation of a Lebesgue measure space $(\Omega, \mathfrak{B}, P), P(\Omega)=1$ with pure point spectra.

(1) The mapping $U \rightarrow \chi_{U}$ is one to one from $C(T)$ onto the group consisting of all homomorphisms from $S_{p}(T)$ into $[0,1)$.

(2) If $\chi_{U}(\gamma)=0$ implies $\gamma=0$, then $U$ is ergodic. 
(3) If there is a homomorphism $\phi$ from $S_{p}(T)$ into $[0,1)$ such that $p \phi(\gamma)$ $=\gamma$ for $\gamma$ in $S_{p}(T)$, then there is a p-th root of $T$.

(4) If there is a 1-parameter group $\phi_{t}$ of homomorphisms from $S_{p}(T)$ into $[0,1)$ such that $\phi_{t}(\gamma)$ is a measurable with respect to $t$ and $\phi_{1}(\gamma)=\gamma$ for $\gamma$ in $S_{p}(T)$, then $T$ is embeddable in a measurable flow.

(5) If there is a homomorphism $\phi$ from $S_{p}(T)$ into $[0,1)$ such that $n \phi(\gamma)$ $=m \gamma$ for $\gamma$ in $S_{p}(T)$ implies $n=m=0$, and such that $\phi(\gamma)=0$ implies $\gamma=0$, then there is an ergodic transformation irrationally commuting with $T$.

Proof. (1) We denote by $\widehat{S_{p}(T)}$ the character group of $S_{p}(T)$ and by $\langle\gamma, g\rangle, \gamma$ in $S_{p}(T), g$ in $\widehat{S_{p}(T)}$ an inner product which is a bilinear form with absolute value 1. Let $a$ be an element in $\widehat{S_{p}(T)}$ defined by $\langle\gamma, a\rangle=\exp (2 \pi i \gamma)$ for $\gamma$ in $S_{p}(T)$ and identify it with the translation $g \rightarrow g+a$ of $S_{p}(T)$. By HalmosNeumann theorem [5] there is a measure preserving mapping $\Psi$ from $(\Omega, \mathfrak{B}, P)$ onto $\widehat{S_{p}(T)}$ with the Haar measure such that $g+a=\Psi T \Psi^{-1} g$ for a.e. $g$ in $\widehat{S_{p}(T)}$. For a homomorphism $\phi$ from $S_{p}(T)$ into $[0,1)$ identify the element $b$ in $\widehat{S_{p}(T)}$ defined by $\langle\gamma, b\rangle=\exp (2 \pi i \phi(\gamma))$ for $\gamma$ in $S_{p}(T)$ with the translation $g \rightarrow g+b$ of $\widehat{S_{p}(T)}$. Then the transformation $U$ of $(\Omega, \mathfrak{B}, P)$ defined by $U \omega=\Psi^{-1} b \Psi \omega$ for $\omega$ in $\Omega$ is in $C(T)$ with $\chi_{U}(\gamma)=\phi(\gamma)$ for $\gamma$ in $S_{p}(T)$. This means the mapping is onto. If $\chi_{U}(\gamma)=0$ for any $\gamma$ in $S_{p}(T)$, then $f_{\gamma}(U \omega)=f_{\gamma}(\omega)$ for every eigenfunction $f_{\gamma}(\omega)$ all of which span $L^{2}(\Omega, P)$. Hence, $U$ is the identity transformation and it follows that the mapping is one to one.

(2) Let $f(\omega)$ be a $U$-invariant function in $L^{2}(\Omega, P)$. Then we have

$$
\begin{aligned}
\int f(\omega) \overline{f_{\gamma}(\omega)} d P(\omega) & =\int f(U \omega) \overline{f_{\gamma}(U \omega)} d P(\omega) \\
& =\overline{\exp \left(2 \pi i \chi_{U}(\gamma)\right)} \int f(\omega) \overline{f_{\gamma}(\omega)} d P(\omega)
\end{aligned}
$$

Since $\chi_{U}(\gamma) \neq 0$ for $\gamma \neq 0, \int f(\omega) \overline{f_{\gamma}(\omega)} d P(\omega)=0$ for $\gamma \neq 0$. Hence, $f(\omega)$ is constant a.e. and $U$ is ergodic.

(3), (4) and (5) follow easily from (1) and Lemma 3.

An infinite sequence $\lambda_{1}, \lambda_{2}, \ldots, \lambda_{n}, \ldots$ of real numbers is called to be rationally independent if for any positive integer $n \quad q_{1} \lambda_{1}+q_{2} \lambda_{2}+\cdots+q_{n} \lambda_{n}=0, q_{i}$ in $\mathbb{Q}, i=1,2, \ldots, n$ implies $q_{1}=q_{2}=\cdots=q_{n}=0$, where $Q$ is the set of all rational numbers.

Theorem 6. Let $T$ be an ergodic measure preserving transformation of a Lebesgue space $(\Omega, \mathfrak{B}, P), P(\Omega)=1$ with pure point spectrum. 
(1) If there is a rationally independent sequence $1, \lambda_{1}, \lambda_{2}, \ldots, \lambda_{n}, \ldots$, such that $S_{p}(T) \subset\left\{m+q_{1} \lambda_{1}+q_{2} \lambda_{2}+\cdots+q_{n} \lambda_{n}: n \in \mathbb{N}, m \in \mathbb{Z}, q_{i} \in \mathbb{Q}\right\}$, then $T$ is embeddable in a measurable flow.

(2) If $n / p$ is not in $S_{p}(T)$ for $n=1,2, \ldots, p-1$, then there is a $p$-th root of $T$.

Proof. (1) Define $\phi_{t}(\gamma)=t\left(q_{1} \lambda_{1}+q_{2} \lambda_{2}+\cdots+q_{n} \lambda_{n}\right)(\bmod 1)$ for $\gamma=$ $m+q_{1} \lambda_{1}+q_{2} \lambda_{2}+\cdots+q_{n} \lambda_{n}$ and $-\infty<t<+\infty$, then $\left\{\phi_{t}\right\}$ is a 1-parameter measurable group of homomorphisms from $S_{p}(T)$ into $[0,1)$ with $\phi_{1}(\gamma)=\gamma$ for $\gamma$ in $S_{p}(T)$. Hence, by Lemma 5 (4) $T$ is embeddable in a measurable flow.

(2) We show first a proof of (2) in case of prime $p$ (Blum and Friedman [2]) for completeness of the discussion, and next, one in case of not prime $p$.

Let $\Gamma$ be a maximal subgroup of $[0,1)$ such that $\Gamma$ includes $S_{p}(T)$ and that $n / p$ is not in $\Gamma$ for $n=1,2, \ldots, p-1$. We show that there exists a homomorphism $\phi$ from $\Gamma$ into itself such that $p \phi(\gamma)=\gamma$ for $\gamma$ in $\Gamma$. It is enough to see that for any $\gamma$ in $\Gamma$ there exists uniquely an integer $n, n=0,1, \ldots, p-1$ such that $(\gamma+n) / p$ is in $\Gamma$. If there is $\gamma$ in $\Gamma$ such that $(\gamma+n) / p$ is not in $\Gamma$ for any $n=0,1, \ldots, p-1$, then $\{m \gamma / p+\eta: m \in \mathbb{Z}, \eta \in \Gamma\}$ is a subgroup of $[0,1)$ including $\Gamma$ and does not contain $n / p$ for any $n=1,2, \ldots, p-1$. This contradicts to the maximality of $\Gamma$. The uniqueness follows easily from that $n / p$ is not in $\Gamma$ for any $n=1,2, \ldots$, $p-1$.

Let $p=p_{1} p_{2} \cdots p_{k}$, where $p_{i}$ 's are prime numbers. Since $1 / p_{1}$ is not in $S_{p}(T)$, from the above discussion there is a homomorphism $\phi_{1}$ from $S_{p}(T)$ into $[0,1)$ such that $p_{1} \phi_{1}(\gamma)=\gamma$ for $\gamma$ in $S_{p}(T)$. Denote by $\Gamma_{1}$ the $\phi_{1}$-image of $S_{p}(T)$. If $\phi_{1}(\gamma)=1 / p_{2}$ for some $\gamma$ in $S_{p}(T)$, then $\gamma=p_{1} \phi_{1}(\gamma)=p_{1} / p_{2}$, which contradicts the assumption of $S_{p}(T)$ if $p_{1} \neq p_{2}$, and $\gamma \neq 0$ if $p_{1}=p_{2}$, respectively. Therefore, $1 / p_{2}$ is not in $\Gamma_{1}$. By the same way we can obtain a sequence $\phi_{2}, \phi_{3}, \ldots, \phi_{k}$ of homomorphisms and a sequence $\Gamma_{2}, \Gamma_{3}, \ldots, \Gamma_{k}$ of subgroups of $[0,1)$ such that $\phi_{i}$ is a homomorphism from $\Gamma_{i-1}$ onto $\Gamma_{i}$ and $p_{i} \phi_{i}(\gamma)=\gamma$ for $\gamma$ in $\Gamma_{i-1}$, for $i=2,3, \ldots, k$. Define a homomorphism $\phi$ from $S_{p}(T)$ into $[0,1)$ by $\phi(\gamma)$ $=\phi_{k}\left(\cdots\left(\phi_{3}\left(\phi_{2}\left(\phi_{1}(\gamma)\right) \cdots\right)\right)\right)$ for $\gamma$ in $S_{p}(T)$. Then we have $p \phi(\gamma)=\gamma$ for $\gamma$ in $S_{p}(T)$ and by Lemma $5(3)$ there is a $p$-th root of $T$.

Lemma \%. Let $p^{\prime}$ and $q^{\prime}$ be positive integers with $\left(p^{\prime}, q^{\prime}\right)=1$, then for a prime number $p$ and a positive integer $q$

$$
\left(q^{\prime}+p^{\prime} q, p^{\prime} p\right)=1 \quad \text { or } \quad\left(q^{\prime}+p^{\prime}(q+1), p^{\prime} p\right)=1 .
$$


Proof. Assume that $a=\left(q^{\prime}+p^{\prime} q, p^{\prime} p\right)>1$ and $a^{\prime}=\left(p^{\prime}, a\right)>1$. Then since $a$ is a divisor of $q^{\prime}+p^{\prime} q, a^{\prime}$ is a divisor of $q^{\prime}$, and hence, $\left(p^{\prime}, q^{\prime}\right) \geqq a^{\prime}>1$, contradiction. Thus $\left(q^{\prime}+p^{\prime} q, p^{\prime} p\right)>1$ implies that $\left(p^{\prime}, a\right)=1$, that $p=a=\left(q^{\prime}+p^{\prime} q\right.$, $\left.p^{\prime} p\right)$ because $p$ is prime, and hence, that $p$ is a divisor of $q^{\prime}+p^{\prime} q$ but not of $p^{\prime}$. Therefore the assumption that $\left(q^{\prime}+p^{\prime} q, p^{\prime} p\right)>1$ and $\left(q^{\prime}+p^{\prime}(q+1), p^{\prime} p\right)>1$ implies that $p$ is a divisor both of $q^{\prime}+p^{\prime} q$ and $q^{\prime}+p^{\prime}(q+1)$ but not of $p^{\prime}$. It is impossible. This proves the lemma.

Theorem 8. For an ergodic measure preserving $T$ with pure point spectra there exists an ergodic measure preserving transformation which commutes irrationally with $T$.

Proof. By Lemma 5 (5) it is enough to construct a homomorphism $\phi$ from $S_{p}(T)$ into $[0,1)$ such that $\phi(\gamma)=0$ implies $\gamma=0$ and such that $l \phi(\gamma)=m \gamma$ for $\gamma$ in $S_{p}(T)$ implies $l=m=0$.

(1) First we construct it in case that $S_{p}(T) \subset Q$. In this case there is a sequence $p_{0}, p_{1}, p_{2}, \ldots, p_{n}, \ldots$ of prime integers such that $S_{p}(T)=\left\{m / p_{0} p_{1} \cdots p_{n}\right.$ : $n \in \boldsymbol{N}, m \in \boldsymbol{Z}\}$. Let $l_{1}, l_{2}, \ldots, l_{n}, \ldots$ be a sequence of positive integers in which every positive integer appears infinitely often.

(1-1) In case that there are infinitely many distinct prime numbers in the sequence $p_{0}, p_{1}, \ldots, p_{n}, \ldots$ Let $n_{0}=0$ and $q_{0}=1$. Having chosen $n_{0}, n_{1}, \ldots$, $n_{k-1}$ and $q_{0}, q_{1}, \ldots, q_{n_{k-1}}, n_{k}$ can be chosen such that there is an integer $q$ with $1 / 3 l_{k}<(q-1) / p_{n_{k}}<(q+1) / p_{n_{k}}<2 / 3 l_{k}$, and $q_{i}$ for $i=n_{k-1}+1, n_{k-1}+2, \ldots, n_{k}-1$, $n_{k}$ can be chosen by Lemma 7 such that $\left(q_{0}+p_{0} q_{1}+\cdots+p_{0} p_{1} \cdots p_{i-1} q_{i}, p_{0} p_{1} \cdots p_{i}\right)$ $=1$ for $n_{k-1}+1 \leqq i \leqq n_{k}$ and $1 / 3 l_{k}<q_{n_{k}} / p_{n_{k}}<\left(q_{n_{k}}+1\right) / p_{n_{k}}<2 / 3 l_{k}$. For the obtained $q_{0}, q_{1}, \ldots, q_{n}, \ldots$ we define

$$
\phi\left(m / p_{0} p_{1} \cdots p_{n}\right)=m\left(q_{0}+p_{0} q_{1}+\cdots+p_{0} p_{1} \cdots p_{n-1} q_{n}\right) / p_{0} p_{1} \cdots p_{n} \quad(\bmod 1) .
$$

Then $\phi$ is a homomorphism from $S_{p}(T)$ into $[0,1)$. If $\phi\left(m / p_{0} p_{1} \cdots p_{n}\right)=0$ $(\bmod 1)$, then from $\left(q_{0}+p_{0} q_{1}+\cdots+p_{0} p_{1} \cdots p_{n-1} q_{n}, p_{0} p_{1} \cdots p_{n}\right)=1 m$ is a multiple of $p_{0} p_{1} \cdots p_{n}$, and hence, $m / p_{0} p_{1} \cdots p_{n}=0(\bmod 1)$. For a positive integer $l$ and infinitely many positive integers $k$ such that $l_{k}=l$ we have $1 / 3<l \phi\left(1 / p_{0} p_{1} \cdots p_{n_{k}}\right)$ $=l_{k}\left(q_{0}+p_{0} q_{1}+\cdots+p_{0} p_{1} \cdots p_{n_{k}-1} q_{n_{k}}\right) / p_{0} p_{1} \cdots p_{n_{k}}<2 / 3$. Since, on the other hand, $m / p_{0} p_{1} \cdots p_{n_{k}}$ converges to 0 as $k \rightarrow \infty$ for an integer $m, l \phi(\gamma)=m \gamma(\bmod 1)$ for $\gamma$ in $S_{p}(T)$ does not hold for positive integers $l$ and $m$.

(1-2) In case that there are only finite number of distinct prime numbers in the sequence $p_{0}, p_{1}, \ldots, p_{n}, \ldots$, we may assume that $S_{p}(T)=\left\{s / p_{0}^{n}+t / p_{1} p_{2} \cdots p_{m}\right.$ : 
$n, m \in \mathbb{N}, s, t \in \mathbb{Z}\}$, where $p_{0}$ does not appear in the sequence $p_{1}, p_{2}, \ldots, p_{n}, \ldots$ Let $n_{0}=0$ and $q_{0}=1$. Having chosen $n_{0}, n_{1}, \ldots, n_{k-1}$ and $q_{0}, q_{1}, \ldots, q_{k-1}$, we take $n_{k}\left(>n_{h-1}\right)$ and $q_{h}$ such that $1 / 3 l_{k}<q_{k} / p_{0}^{n_{k}-n_{k-1}}<\left(q_{k}+1\right) / p_{0}^{n_{k}-n_{k-1}}<2 / 3 l_{h}$. For the obtained $n_{0}, n_{1}, \ldots, n_{h}, \ldots$ and $q_{0}, q_{1}, \ldots, q_{n}, \ldots$ define

$$
\begin{aligned}
& \phi\left(s / p_{0}^{n_{k}}+t / p_{1} p_{2} \cdots p_{m}\right) \\
& \quad=s\left(q_{0}+p_{0}^{n_{1}} q_{1}+\cdots+p_{0}^{n_{k-1}} q_{k}\right) / p_{0}^{n_{k}}+t / p_{1} p_{2} \cdots p_{m} \quad(\bmod 1) .
\end{aligned}
$$

Then $\phi$ is a homomorphism from $S_{p}(T)$ into $[0,1)$. From $q_{0}=1,\left(q_{0}+p_{0}^{n_{1}} q_{1}\right.$ $\left.+\cdots+p_{0}^{n_{h}-1} q_{h}, p_{0}^{n_{k}}\right)=1$, and hence, $\phi(\gamma)=0$ implies $\gamma=0$. Since $1 / 3<l_{h}\left(q_{0}\right.$ $\left.+p_{0}^{n_{1}} q_{1}+\cdots+p_{0}^{n_{k}-1} q_{k}\right) / p_{0}^{n_{h}}<2 / 3$, by the same way as one in $(1-1)$ we can show that $l \phi(\gamma)=m \gamma$, for $\gamma$ in $S_{p}(T)$ holds only for $l=m=0$.

(2) In case that an irrational number $\lambda_{1}$ is in $S_{p}(T)$, there is a rationally independent sequence $1, \lambda_{1}, \lambda_{2}, \ldots, \lambda_{m}, \ldots$ such that any number in $S_{p}(T)$ has a form $q_{0}+q_{1} \lambda_{1}+\cdots+q_{n} \lambda_{n}, n \in \mathbb{N}, q_{i} \in \boldsymbol{Q}, i=0,1, \ldots, n$. Let $1, \eta_{1}, \eta_{2}, \ldots, \eta_{n}, \ldots$ be another rationally independent sequence such that $\eta_{1}$ and $\lambda_{1}$ are rationally independent and define

$$
\phi\left(q_{0}+q_{1} \lambda_{1}+\cdots+q_{n} \lambda_{n}\right)=q_{0}+q_{1} \eta_{1}+\cdots+q_{n} \eta_{n}(\bmod 1), \quad n \in \mathbb{N}, q_{i} \in \mathbb{Q},
$$

$i=0,1, \ldots, n$. Then $\phi$ is a homomorphism from $S_{p}(T)$ into $[0,1)$. It is obvious that $\phi(\gamma)=0$ implies $\gamma=0$. If $l \phi(\gamma)=m \gamma$ for $\gamma$ in $S_{p}(T)$, we have $l \phi\left(\lambda_{1}\right)=\ln \eta_{1}$ $=m \eta_{1}$ and $l=m=0$ follows from that $\lambda_{1}$ and $\eta_{1}$ are rationally independent. The proof is complete.

\section{§3. Examples}

Let $\Gamma$ be a torsionfree countable abelian group and $G$ the character group of $\Gamma$ which is a separable compact abelian group. We denote by $\langle\gamma, g\rangle, \gamma \in \Gamma$, $g \in G$ their inner product and by $d g$ the Haar measure on $G$. We note that an endomorphism (automorphism) $\sigma$ of $G$ determines uniquely an endomorphism (automorphism, respectively) $\hat{\sigma}$ of $\Gamma$ by $\langle\gamma, \sigma(g)\rangle=\langle\hat{\sigma}(\gamma), g\rangle, \gamma \in \Gamma, g \in G$, and vice versa. We denote by $\operatorname{End}(G)$ and $\operatorname{End}(\Gamma)$ the sets of all endomorphisms of $G$ and $\Gamma$, respectively.

Theorem 9. For elements $\lambda$ and $\eta$ in $G$ and $\sigma$ in $\operatorname{End}(G)$ let $U_{\sigma, \lambda, \eta}$ be a measure preserving transformation of the direct product measure space $(G \times G$, $d g \times d g)$ defined by $U_{\sigma, \lambda, \eta}\left(g, g^{\prime}\right)=\left(g+\lambda, g^{\prime}+\sigma(g)+\eta\right)$ for $\left(g, g^{\prime}\right)$ in $G \times G$. If $\langle\gamma, \lambda\rangle=1$ implies $\gamma=0$ and if $\hat{\sigma}(\gamma)=0$ implies $\gamma=0$, then (1) $U_{\sigma . \lambda, \eta}$ is ergodic, 
(2) $\exp \left(2 \pi i S_{p}\left(U_{\sigma, \lambda, \eta}\right)\right)=\{\langle\gamma, \lambda\rangle: \gamma \in \Gamma\}$ and (3) $C\left(U_{\sigma, \lambda, \eta}\right)=\left\{U_{\delta, \alpha, \beta}: \delta \in\right.$ End $(G)$, $\alpha, \beta \in G$ with $\sigma(\alpha)=\delta(\lambda)\}$, where the left side of (2) is the set consisting of all $\exp (2 \pi i \zeta) \operatorname{sor} \zeta$ in $S_{p}\left(U_{\sigma, \lambda, \eta}\right)$.

Proof. Since $\left\{\langle\gamma, g\rangle\left\langle\gamma^{\prime}, g^{\prime}\right\rangle: \gamma, \gamma^{\prime} \in \Gamma\right\}$ is a complete orthonormal system of $L^{2}(G \times G, d g \times d g)$, any function in it is represented as $f\left(g, g^{\prime}\right)=\sum_{\gamma, \gamma^{\prime}} a_{\gamma, \gamma^{\prime}}$ $\langle\gamma, g\rangle\left\langle\gamma^{\prime}, g^{\prime}\right\rangle$ for $\left(g, g^{\prime}\right)$ in $G \times G$ and we have $f\left(U_{\sigma . \lambda, \eta}\left(g, g^{\prime}\right)\right)=\sum_{\gamma, \gamma^{\prime}} a_{\gamma, \gamma^{\prime}}$ $\langle\gamma, \lambda\rangle\left\langle\gamma^{\prime}, \eta\right\rangle\left\langle\gamma+\hat{\sigma}\left(\gamma^{\prime}\right), g\right\rangle\left\langle\gamma^{\prime}, g^{\prime}\right\rangle$ for $\left(g, g^{\prime}\right)$ in $G \times G$.

(1) From $f\left(U_{\sigma, \lambda, \eta}\left(g, g^{\prime}\right)\right)=f\left(g, g^{\prime}\right)$ for $\left(g, g^{\prime}\right)$ in $G \times G$ we have $a_{\gamma, \gamma^{\prime}}\langle\gamma, \lambda\rangle$ $\left\langle\gamma^{\prime}, \eta\right\rangle=a_{\gamma+\hat{\sigma}\left(\gamma^{\prime}\right), \gamma^{\prime}}$ for $\gamma, \gamma^{\prime}$ in $\Gamma$. Hence, $\left|a_{\gamma, \gamma^{\prime}}\right|=\left|a_{\gamma+\hat{\sigma}\left(\gamma^{\prime}\right), \gamma^{\prime}}\right|=\left|a_{\gamma+2 \hat{\sigma}\left(\gamma^{\prime}\right), \gamma^{\prime}}\right|=\cdots$. Since $f\left(g, g^{\prime}\right)$ is in $L^{2}(G \times G, d g \times d g), \Gamma$ is torsionfree and since $\hat{\sigma}(\gamma)=0$ implies $\gamma=0$, we have $a_{\gamma, \gamma^{\prime}}=0$ for $\gamma^{\prime} \neq 0$. Since $a_{\gamma, 0}\langle\gamma, \lambda\rangle=a_{\gamma, 0}$ and since $\langle\gamma, \lambda\rangle=1$ implies $\gamma=0$ we have $a_{\gamma, 0}=0$ for $\gamma \neq 0$. Hence, $f\left(g, g^{\prime}\right)=a_{0,0}$ and $U_{\sigma, \lambda, \eta}$ is ergodic.

(2) Let $f\left(g, g^{\prime}\right)$ be an eigenfunction of $U_{\sigma, \lambda, \eta}$ for a point spectrum $\zeta$, then we have $a_{\gamma, \gamma^{\prime}}\langle\gamma, \lambda\rangle\left\langle\gamma^{\prime}, \eta\right\rangle=\exp (2 \pi i \zeta) a_{\gamma+\hat{\sigma}\left(\gamma^{\prime}\right), \gamma^{\prime}}, \gamma, \gamma^{\prime} \in \Gamma$. By the same way as one in (1) we have $a_{\gamma, \gamma^{\prime}}=0$ for $\gamma^{\prime} \neq 0$. Since $f\left(g, g^{\prime}\right)$ is not zero function there is $\gamma$ with $a_{\gamma, 0} \neq 0$. For such $\gamma$ it follows from $a_{\gamma, 0}\langle\gamma, \lambda\rangle=\exp (2 \pi i \zeta) a_{\gamma, 0}$ that $\langle\gamma, \lambda\rangle=\exp (2 \pi i \zeta)$, which means $\exp \left(2 \pi i S_{p}\left(U_{\sigma, \lambda, \eta}\right)\right)=\{\langle\gamma, \lambda\rangle: \gamma \in \Gamma\}$. It is obvious that every $\langle\gamma, \lambda\rangle$ is in $\exp \left(2 \pi i S_{p}\left(U_{\sigma, \lambda, \eta}\right)\right)$.

(3) Let $V$ be a transformation in $C\left(U_{\sigma, \lambda, \eta}\right)$ and put $f_{\gamma}\left(g, g^{\prime}\right)=\langle\gamma, g\rangle$ for $\gamma$ in $\Gamma$ and $\left(g, g^{\prime}\right)$ in $G \times G$. Since $f_{\gamma}$ is an eigenfunction of $U_{\sigma, \lambda, \eta}$, by Lemma 1 there is an element $\alpha$ in $G$ such that $f_{\gamma}\left(V\left(g, g^{\prime}\right)\right)=\langle\gamma, \alpha\rangle f_{\gamma}\left(g, g^{\prime}\right)$ for $\left(g, g^{\prime}\right)$ in $G \times G$. From $f_{\gamma}\left(V\left(g, g^{\prime}\right)\right)=\langle\gamma, g+\alpha\rangle$ there is a mapping $\Phi$ from $G \times G$ onto $G$ such that $V\left(g, g^{\prime}\right)=\left(g+\alpha, \Phi\left(g, g^{\prime}\right)\right)$ for $\left(g, g^{\prime}\right)$ in $G \times G$. From $V U_{\sigma, \lambda, \eta}=U_{\sigma, \lambda, \eta} V$ we have $\Phi\left(U_{\sigma, \lambda, \eta}\left(g, g^{\prime}\right)\right)=\Phi\left(g, g^{\prime}\right)+\sigma(g+\alpha)+\eta$ for $\left(g, g^{\prime}\right)$ in $G \times G$. Put $\Psi\left(g, g^{\prime}\right)=$ $\Phi\left(g, g^{\prime}\right)-g^{\prime}$, then $\Psi\left(U_{\sigma, \lambda, \eta}\left(g, g^{\prime}\right)\right)=\Psi\left(g, g^{\prime}\right)+\sigma(\alpha)$ for $\left(g, g^{\prime}\right)$ in $G \times G$. Hence, $\langle\gamma, \sigma(\alpha)\rangle$ is in $\exp \left(2 \pi i S_{p}\left(U_{\sigma, \lambda, \eta}\right)\right)$ for any $\gamma$ in $\Gamma$. By (2) there is an element $\hat{\sigma}(\gamma)$ in $\Gamma$ such that $\langle\gamma, \sigma(\alpha)\rangle=\langle\hat{\delta}(\gamma), \lambda\rangle$ for $\gamma$ in $\Gamma$. From the property of $\lambda, \hat{\delta}$ is an endomorphism of $\Gamma$ and $\sigma(\alpha)=\delta(\lambda)$. Put $\Theta\left(g, g^{\prime}\right)=\Phi\left(g, g^{\prime}\right)-g^{\prime}-\delta(g)$ for $\left(g, g^{\prime}\right)$ in $G \times G$, we have $\Theta\left(U_{\sigma, \lambda, \eta}\left(g, g^{\prime}\right)\right)=\Theta\left(g, g^{\prime}\right)$ for $\left(g, g^{\prime}\right)$ in $G \times G$. From (1) there is an element $\beta$ in $G$ such that $\Theta\left(g, g^{\prime}\right)=\beta$ for $\left(g, g^{\prime}\right)$ in $G \times G$. Hence, $V\left(g, g^{\prime}\right)=\left(g+\alpha, g^{\prime}+\delta(g)+\beta\right)$ for $\left(g, g^{\prime}\right)$ in $G \times G$, that is, $V=U_{\delta, \alpha, \beta}$. Conversely, $U_{\delta, \alpha, \beta}$ with $\sigma(\alpha)=\delta(\lambda)$ is in $C\left(U_{\sigma, \lambda, \eta}\right)$.

For a sequence $p_{1}, p_{2}, \ldots, p_{n}, \ldots$ of prime numbers let $Q_{0}$ be the subgroup of $\mathbb{Q}$ consisting of all rational numbers of the form $m / p_{1} p_{2} \cdots p_{n}, n \in \mathbb{N}, m \in \mathbb{Z}$, 
and $\Omega$ be the infinite direct product set $[0,1) \times \prod_{n=1}^{\infty}\left\{0,1, \ldots, p_{n}-1\right\}$. Define $\langle q, \omega\rangle$ for $q=m / p_{1} p_{2} \cdots p_{n}$ in $\mathbb{Q}_{0}$ and $\omega$ in $\Omega$ by $\langle q, \omega\rangle=\exp \left(2 \pi i m\left(\omega_{0}+\omega_{1}\right.\right.$ $\left.\left.+p_{1} \omega_{2}+\cdots+p_{1} p_{2} \cdots p_{n-1} \omega_{n}\right) / p_{1} p_{2} \cdots p_{n}\right)$, where $\omega_{n}$ is the $n$-th coordinate of $\omega$. Then $\Omega$ is the dual of $Q_{0}$ with respect to the inner product $\langle q, \omega\rangle$. The adding operation in $\Omega$ defined by $\left\langle q, \omega+\omega^{\prime}\right\rangle=\langle q, \omega\rangle\left\langle q, \omega^{\prime}\right\rangle, q \in \mathbb{Q}_{0}, \omega, \omega^{\prime} \in \Omega$ may be called a generalized adding machine. The Haar measure $P$ on $\Omega$ is the infinite direct product $d \omega_{0} \times \prod_{n=1}^{\infty}\left\{1 / p_{n}, 1 / p_{n}, \ldots, 1 / p_{n}\right\}$. Let $\mathbb{Q}_{1}$ be the set of all rational numbers of the form $m / p(1)^{n(1)} p(2)^{n(2)} \cdots p(k)^{n(k)}, k \in \mathbb{N}, m \in \mathbb{Z}$, $n(i) \in \mathbb{N}$ and $p(i)$ 's are prime numbers each of which appears infinitely many times in the sequence $p_{1}, p_{2}, \ldots, p_{n}, \ldots$. For a number $q_{1}$ in $\mathbb{Q}_{1}$ a mapping $q \rightarrow$ $q_{1} q$ is an endomorphism of $\mathbb{Q}_{0}$ and so, for $q_{1}$ in $\mathbb{Q}_{1}$ and $\omega$ in $\Omega, q_{1} \omega$ is defined such that $\omega \rightarrow q_{1} \omega$ is an endomorphism of $\Omega$. We can see that there are no other endomorphisms of $\mathbb{Q}_{0}$ (or $\Omega$ ) except ones determined by numbers in $\mathbb{Q}_{1}$ as above. Let $\mathbb{Q}_{2}$ be the set of all numbers $q_{1}$ in $Q_{1}$ such that $1 / q_{1}$ is also in $\mathbb{Q}_{1}$, then the endomorphism of $Q_{0}$ (or $\Omega$ ) determined by $q_{1}$ in $Q_{1}$ is an automorphism if and only if $q_{1}$ is in $Q_{2}$.

Theorem 10. Let $\Omega$ be the compact abelian group described above and $\lambda$ be an element of $\Omega$ whose 0 -th coordinate $\lambda_{0}$ is irrational. Define a measure preserving transformation $T$ of the direct product space $(\Omega \times \Omega, P \times P)$ by $T\left(\omega, \omega^{\prime}\right)=\left(\omega+\lambda, \omega^{\prime}+\omega\right)$ for $\left(\omega, \omega^{\prime}\right)$ in $\Omega \times \Omega$. Then we have the followings:

(1) $T$ is ergodic.

(2) $C(T)=\left\{U_{q_{1}, \alpha}: q_{1} \in \mathbb{Q}_{1}, \alpha \in \Omega\right\}$, where $U_{q_{1}, \alpha}\left(\omega, \omega^{\prime}\right)=\left(\omega+q_{1} \lambda, \omega^{\prime}+q_{1} \omega+\alpha\right)$ for $\left(\omega, \omega^{\prime}\right)$ in $\Omega \times \Omega$.

(3) $T$ is not embeddable in a measurable flow.

(4) If $U_{q_{1}, \alpha}^{p}=T^{m}, p, m \in Z$, then $m=p q_{1}$ and

$$
p \alpha=m(m-1) \lambda / 2-p(p-1) q_{1}^{2} \lambda / 2 .
$$

(5) If there is a $p$-th root of $T, p$ is in $\mathbb{Q}_{2}$.

(6) There is an ergodic measure preserving transformation which commutes irrationally with $T$.

Proof. (1) and (2) follow from Theorem 9.

(3) Let $\left\{U_{t}\right\}$ be a measurable flow in $C(T)$, then by (2) we may assume $U_{t}=U_{q_{1}(t), \alpha(t)}$ for $q_{1}(t)$ in $Q_{1}$ and $\alpha(t)$ in $\Omega$. Since $\omega \rightarrow \omega+q_{1}(t) \lambda$ is a measurable flow of $\Omega, q_{1}(t)=0$ follows from the countability of $\mathbb{Q}_{1}$. Thus we conclude that $U_{1}=U_{0, \alpha(1)} \neq T$ for any measurable flow $\left\{U_{t}\right\}$ in $C(T)$. 
(4) follows from $T^{m}\left(\omega, \omega^{\prime}\right)=\left(\omega+m \lambda, \omega^{\prime}+m \omega+m(m-1) \lambda / 2\right)$ and $U_{q_{1}, \alpha}^{p}\left(\omega, \omega^{\prime}\right)=\left(\omega+p q_{1} \lambda_{,}, \omega^{\prime}+p q_{1} \omega+p \alpha+p(p-1) q_{1}^{2} \lambda / 2\right),\left(\omega, \omega^{\prime}\right) \in \Omega \times \Omega$.

(5) Let $U=U_{q_{1}, \alpha}$ is a $p$-th root of $T$ then by (4) we have $p q_{1}=1$, which implies that $p$ is in $\boldsymbol{Q}_{2}$.

(6) Let $U=U_{q_{1}, \alpha}$ for $q_{1} \neq 0$ in $\boldsymbol{Q}_{1}$ and $\alpha$ in $\Omega$ such that the 0 -th coordinate $\alpha_{0}$ of $\alpha$ is rationally independent with $\lambda_{0}$. Then by Theorem $9 U$ is ergodic. If $U^{p}=T^{m}$ then by (4) $p \alpha=\left\{m(m-1) / 2-p(p-1) q_{1}^{2} / 2\right\} \lambda$, and hence, $p=m=0$ follows from that $\alpha_{0}$ and $\lambda_{0}$ are rationally independent.

Example 1. For an irrational number $\lambda$ define a transformation $T$ of $[0,1) \times[0,1)$ by

$$
T(x, y)=(x+\lambda, y+x) \quad \text { for }(x, y) \text { in }[0,1) \times[0,1) .
$$

This $T$ is the same transformation as one in Theorem 9 in case of $p_{n}=1$ for every $n \in \boldsymbol{N}$, in which case $\boldsymbol{Q}_{0}=\boldsymbol{Q}_{1}=\boldsymbol{Z}$ and $\boldsymbol{Q}_{2}=\{1\}$. Since $C(T)=\left\{U_{n, \alpha}\right.$ : $n \in \boldsymbol{Z}, \alpha \in[0,1)\}$, where $\left.U_{n, \alpha}(x, y)=(x+n\rangle, y+n x+\alpha\right)$ for $(x, y)$ in $[0,1)$ $\times[0,1)$, it is easy to see that $T$ has no $p$-th root for every $p \geqq 2$. By Theorem 9 $T^{2}$ is also ergodic and $C\left(T^{2}\right)=C(T) \cup\left\{U_{n, \beta}^{\prime}: n \in \mathbb{Z}, \beta \in[0,1)\right\}$, where $U_{n, \beta}^{\prime}(x, y)$ $=(x+n \lambda+1 / 2, y+n x+\beta)$ for $(x, y)$ in $[0,1) \times[0,1)$. Hence, $T^{2}$ has only a square root $T$ but no other root.

Example 2. For the transformation $T$ of Theorem 10 in case of $p_{n}=2$ for every $n$, we have that $\boldsymbol{Q}_{0}=\boldsymbol{Q}_{1}=$ the group of all 2 -adic rational numbers and $\boldsymbol{Q}_{2}=\left\{2^{n}: n \in \boldsymbol{Z}\right\}$. Then $T$ has a $2^{n}$-th root $U_{1 / 2^{n, \alpha}}\left(\alpha=-\left(2^{n}-1\right) \lambda / 2^{2 n+1}\right)$ for every $n \geqq 1$ but no other root.

Example 3. For the transformation $T$ of Theorem 10 in casc that every prime number appears infinitely often in the sequence $p_{1}, p_{2}, \ldots, p_{n}, \ldots, \boldsymbol{Q}_{0}=\boldsymbol{Q}_{1}$ $=\boldsymbol{Q}$ and $\boldsymbol{Q}_{2}=\boldsymbol{Q}-\{0\}$. Then $T$ has a $p$-th root $U_{1 / p, \alpha}\left(\alpha=-(p-1) \lambda / 2 p^{2}\right)$ for every $p \geqq 2$, but is not embeddable in a measurable flow as seen in Theorem 10 .

Example 4. We consider a dual pair $\mathbb{Z}^{2}=\boldsymbol{Z} \times \boldsymbol{Z},[0,1)^{2}=[0,1) \times[0,1)$ with an inner product $\langle(n, m),(x, y)\rangle=\exp (2 \pi i(n x+m y)),(n, m) \in \mathbb{Z}^{2},(x, y)$ $\in[0,1)^{2}$. For irrational numbers $\lambda$ and $\eta$ which are rationally independent define a transformation $T$ of $[0,1)^{4}$ by

$$
T(x, y, z, w)=(x+\lambda, y+\eta, z+x, w+y) \text { for }(x, y, z, w) \text { in }[0,1)^{4} .
$$

Then by Theorem $9 T$ is ergodic, $S_{p}(T)=\left\{n \lambda+m \eta(\bmod 1):(n, m) \in \mathbb{Z}^{2}\right\}$ and $C(T)=\left\{U_{\sigma, \alpha}: \sigma\right.$ is an endomorphism of $S_{p}(T), \alpha$ in $\left.[0,1)^{2}\right\}$, where $U_{\sigma . \alpha}(x, y, z, w)$ 
$=(x+a \lambda+c \eta, y+b \lambda+d \eta, z+a x+c y+e, w+b x+d y+f)$ for $(x, y, z, w)$ in $[0,1)^{4}$ if $\sigma$ is given by $2 \times 2$ matrix $\left(\begin{array}{ll}a & b \\ c & d\end{array}\right)$ with integer components $a, b, c, d$ and $\alpha=(e, f)$. Since $2 \times 2$ matrices are not commutative, $C(T)$ is not commutative. Note that this $T$ is the product transformation of ones of Example 1, whose commutants are commutative.

Example 5. For irrational numbers $\lambda$ and $\eta$ which are rationally independent define a transformation $T$ of $[0,1)^{3}$ by $T(x, y, z)=(x+\lambda, y+\eta$, $x+y+z)$ for $(x, y, z)$ in $[0,1)^{3}$. By the same way as one of Theorem 9 we can see that $T$ is ergodic and $C(T)=\left\{U_{n, m, a, b}: n, m \in \mathbb{Z}, a, b \in[0,1)\right\}$, where $U_{n, m, a, b}(x, y, z)=(x+n \lambda+a, y+m \eta-a, z+n x+m y+b)$ for $(x, y, z)$ in $[0,1)^{3}$. This $C(T)$ is also not commutative.

\section{§4. Application to the Theory of Normalizer of Ergodic Non-singular Transformation}

For an ergodic non-singular transformation $\tau$ of a Lebesgue space $(W, \mathfrak{F}, v)$ we denote by $\operatorname{Orb}_{\tau}(w)$ the orbit $\left\{\tau^{n} w: n \in \mathbb{Z}\right\}$ of $w$ in $W$. The full group $[\tau]$ of $\tau$ is defined as the set consisting of all non-singular transformations $\xi$ of $(W, \mathfrak{F}, v)$ such that $\xi_{w}$ is in $\operatorname{Orb}_{\tau}(w)$ for a.e. $w$ in $W$. A non-singular transformation $R$ of $(W, \mathfrak{F}, v)$ is called a normalizer of $[\tau]$ if $R[\tau] R^{-1}=[\tau]$. An ergodic nonsingular transformation $\tau$ determines uniquely up to conjugacy an ergodic non-singular flow $\left\{A_{t}\right\}$ called the associated flow of $[\tau]$ ([7], [9]) and a normalizer $R$ of $[\tau]$ determines a non-singular transformation $\bmod R$ which commutes with the associated flow $\left\{A_{t}\right\}([8])$. In this paper we call the pair $([\tau], R)$ a non-commutative dynamics with characteristic $\left(\left\{A_{t}\right\}, \bmod R\right)$. T. Hamachi [6] showed that for an ergodic non-singular flow $\left\{T_{t}\right\}$ and a non-singular transformation $U$ in $C\left(\left\{T_{t}\right\}\right)$ there is a non-commutative dynamics with characteristic $\left(\left\{T_{t}\right\}, U\right)$. A non-commutative dynamics $([\tau], R)$ is said to have a $p$-th root if there is a $p$-th root of $R$ which is also a normalizer of $[\tau]$ and to be embeddable in a measurable flow if $R$ is embeddable in a measurable flow which consists of normalizers of $[\tau]$. We can easily see that a non-commutative dynamics $([\tau], R)$ has a $p$-th root (is embeddable in a measurable flow) $\bmod R$ has a $p$-th root in $C\left(\left\{A_{t}\right\}\right)$ (is embeddable in a measurable flow in $C\left(\left\{A_{t}\right\}\right.$ ), respectively).

Let $T$ be an ergodic measure preserving transformation of a Lebesgue space 
$(\Omega, \mathfrak{B}, P)$ with $P(\Omega)=1$ and $c>0$. We define a measurable flow $\left\{\bar{T}_{t}\right\}$ on the product set $\bar{\Omega}_{c}=\Omega \times[0, c)$ by $\bar{T}_{t}(\omega, u)=\left(T^{n} \omega, u+t-c n\right)$ if $c n \leqq u+t<c(n+1)$, $(\omega, u) \in \bar{\Omega}_{c} . \quad\left\{\bar{T}_{t}\right\}$ is ergodic and called the special flow with constant ceiling $c$ with base transformation $T$.

Lemma 11. Let $\left\{\bar{T}_{t}\right\}$ be the special flow with constant ceiling $c>0$ with an ergodic base transformation $T$.

(1) Denote by $C\left(\left\{\bar{T}_{t}\right\}\right)$ the group consisting of all transformations each of which commutes with $\bar{T}_{t}$ for every $t$, then $C\left(\left\{\bar{T}_{t}\right\}\right)=\left\{\bar{T}_{\alpha} \bar{U}: \alpha \in[0,1), U \in C(T)\right\}$, where $\bar{U}(\omega, u)=(U \omega, u)$ for $(\omega, u)$ in $\bar{\Omega}_{c}([8])$.

(2) A measurable flow in $C\left(\left\{\bar{T}_{t}\right\}\right)$ has a form $\bar{T}_{\alpha t} \bar{U}_{t}$, $t$ in $R$, where $\alpha$ is a real number and $\left\{U_{t}\right\}$ is a measurable flow in $C(T)$.

(3) A transformation $\bar{T}_{\alpha} \bar{U}$ in $C\left(\left\{\bar{T}_{t}\right\}\right)$ has a $p$-th root if and only if $T^{n} U$ has a p-th root in $C(T)$ for some $n$.

Proof. (1) Let $\Pi$ be the mapping from $\bar{\Omega}_{c}$ onto $[0, c)$ defined by $\Pi(\omega, u)$ $=u$ for $(\omega, u)$ in $\bar{\Omega}_{c}$ and $L$ a transformation in $C\left(\left\{\bar{T}_{t}\right\}\right)$. Since $\Pi(L(\omega, u))-u$ is a $\left\{\bar{T}_{t}\right\}$-invariant measurable function and $\left\{\bar{T}_{t}\right\}$ is ergodic, there is a constant $\alpha$ in $[0, c)$ such that $\Pi(L(\omega, u))=u+\alpha(\bmod c)$ for $(\omega, u)$ in $\bar{\Omega}_{c}$. Hence, $L$ has the form $L(\omega, u)=(U(\omega, u), u+\alpha)$ for $(\omega, u)$ in $\bar{\Omega}_{c}$. From $\bar{T}_{-\alpha} L(\omega, u)$ $=(U(\omega, u), u)$ for $(\omega, u)$ in $\bar{\Omega}_{c}$ and $\bar{T}_{t}\left(\bar{T}_{-\alpha} L\right)=\left(\bar{T}_{-\alpha} L\right) \bar{T}_{t}$ for $t$ in $\mathbb{R}$ follows that $U(\omega, u+t)=U(\omega, u)$ if $0 \leqq u+t<c$, so that $U(\omega, u)$ does not depend on $u$ in $[0, c)$. From $\bar{T}_{c}\left(\bar{T}_{-\alpha} L\right)=\left(\bar{T}_{-\alpha} L\right) \bar{T}_{c}$ we have $U(T \omega)=T U(\omega)$, that is, $U$ is in $C(T)$. We have $L=\bar{T}_{-\alpha} \bar{U}$.

(2) Let $L_{t}$ be a measurable flow in $C\left(\left\{\bar{T}_{t}\right\}\right)$, then by (1) $L_{t}$ has the form $L_{t}=\bar{T}_{\alpha(t)} \bar{U}_{t}$, where for each $t, U_{t}$ is in $C(T)$ and $\alpha(t)$ is a real number. Since $\alpha(t)=\Pi\left(L_{t}(\omega, u)\right)-\Pi(\omega, u)$ is a measurable function and $\alpha(t+s)=\alpha(t)+\alpha(s)$ $(\bmod c)$ for $t, s$ in $R$ there is a real number $\alpha$ with $\alpha(t)=\alpha t(\bmod c)$ for $t$ in $\mathbb{R}$. Since $\bar{U}_{t}=\bar{T}_{-\alpha t} L_{t}, t$ in $\mathbb{R}$ is a measurable flow, so is $\left\{U_{t}\right\}$.

(3) Let $\bar{T}_{\beta} \bar{V}$ be a $p$-th root of $\bar{T} \bar{U}$, where $U$ is in $C(T)$ and $\beta$ is in $[0,1)$. From $\bar{T}_{p \beta} \bar{V}^{p}=\bar{T}_{\alpha} \bar{U}$ there is an integer $n$ such that $p \beta=\alpha-c n$ and $V^{p}=T^{n} U$. Conversely, let $V$ be a transformation in $C(T)$ such that $V^{p}=T^{n} U$ for some integer $n$ and $\beta=(\alpha-c n) / p$, then $\bar{T}_{\beta} \bar{V}$ is a $p$-th root of $\bar{T}_{\alpha} \bar{U}$.

Example 6. For an irrational number $\lambda$ define transformations $T$ and $U$ of $[0,1)^{2}$ by

$$
T(x, y)=(x+2 \lambda, y+2 x) \text { and } U(x, y)=(x+\lambda, y+x) \text { for }(x, y) \text { in }[0,1)^{2} .
$$


As seen in Example $1 T$ is ergodic and $U$ is in $C(T)$. Since $T^{n} U(x, y)=$ $\left(x+(2 n+1) \lambda, y+(2 n+1) x+2 n^{2} \lambda\right)$ for $(x, y)$ in $[0,1)^{2}$ and $n$ in $Z$, by Theorem 9 we have $C\left(T^{n} U\right)=\left\{U_{k, j, \beta}: k \in \mathbb{Z}, j=0,1, \ldots, 2 n, \beta \in[0,1)\right\}$ for $n$ in $\mathbb{Z}$, where $U_{k, j, \beta}(x, y)=(x+k \lambda+j /(2 n+1), y+k x+\beta)$ for $(x, y)$ in $[0,1)^{2}$. Hence, every $T^{n} U$ for $n$ in $\mathbb{Z}$ does not have a square root. Therefore, by Lemma 11 (3) $\bar{U}$ does not have a square root in $C\left(\left\{\bar{T}_{t}\right\}\right)$. Then a non-commutative dynamics with characteristic $\left(\left\{\bar{T}_{t}\right\}, \bar{U}\right)$ has no square root.

Example 7. For a sequence $p_{1}, p_{2}, \ldots, p_{n}, \ldots$ of prime numbers in which every prime number appears infinitely many times let $\Omega=[0,1) \times \prod_{n=1}^{\infty}\{0,1, \ldots$, $\left.p_{n}-1\right\}$ be the compact abelian group as same as one in Theorem 10. For an element $\lambda$ in $\Omega$ such that $\langle\gamma, \lambda\rangle=1$ implies $\gamma=0$ define transformations $T$ and $U$ of $\Omega \times \Omega$ by $T\left(\omega, \omega^{\prime}\right)=\left(\omega+2 \lambda, \omega^{\prime}+2 \omega\right)$ and $U\left(\omega, \omega^{\prime}\right)=\left(\omega+\lambda, \omega^{\prime}+\omega\right)$ for $\left(\omega, \omega^{\prime}\right)$ in $\Omega \times \Omega$. Then by Theorem $10 T$ is ergodic and $U$ in $C(T)$. By the same discussion as one in the proof of Theorem 10 (3) a measurable flow in $C(T)$ has the form $U_{\alpha(t)}\left(\omega, \omega^{\prime}\right)=\left(\omega, \omega^{\prime}+\alpha(t)\right)$, where $\alpha(t)$ is a 1-parameter subgroup of $\Omega$. Hence, by Lemma 11 (2) a measurable flow in $C\left(\left\{\bar{T}_{t}\right\}\right)$ has the form $\bar{T}_{\beta t} \bar{U}_{\alpha(t)}\left(\omega, \omega^{\prime}, u\right)=\left(\omega+2 n \lambda, \omega^{\prime}+2 n \omega+2 n(n-1) \lambda+\alpha(t), u+\beta t-c n\right)$ if $c n$ $\leqq u+\beta t<c(n+1)$, where $\beta$ is a constant. Since $\lambda \neq 2 n \lambda$ for any integer $n, \bar{U}$ $\neq \bar{T}_{\beta t} \bar{U}_{\alpha(t)}$ for any $t$, that is, $\bar{U}$ which is in $C\left(\left\{\bar{T}_{t}\right\}\right)$ is not embeddable in a measurable flow in $C\left(\left\{\bar{T}_{t}\right\}\right)$. Since $U$ has a $p$-th root in $C(T)$ for every $p \geqq 2$ as seen in Example 3, there exists a non-commutative dynamics with characteristic $\left(\left\{\bar{T}_{t}\right\}, \bar{U}\right)$ which has a $p$-th root for every $p \geqq 2$ but which is not embeddable in a measurable flow.

\section{References}

[1] Blum, J. R. and Friedman, N., On roots of transformations, Z. Wahrscheinlichkeitstheorie und Verw. Gebiete, 5 (1966), 1-5.

[2] Blum, J. R. and Friedman, N., On commuting transformations and roots, Proc. Amer. Math. Soc., $\mathbb{1 7}$ (1966), 1370-1374.

[3] Chacon, R. V., Transformations having continuous spectrum, J. of Math. Mech., 16 (1966), 399-415.

[4] Chacon, R. V., A geometric construction of measure preserving transformations, Proc. 5th Berkeley Symp. Math. Statist. Probability, 2 (1967), 335-350.

[ 5 ] Halmos, P. R. and von Neumann, J., Operator methods in classical mechanics II, Ann. of Math., 43 (1942), 332-350.

[6] Hamachi, T., The normalizer group of an ergodic automorphism of type III and the commutant of an ergodic flow, J. Functional Analysis, 40 (1981), 387-403.

[ 7 ] Hamachi, T., Oka, Y. and Osikawa, M., Flows associated with ergodic non-singular transformation groups, Publ. RIMS, Kyoto Univ., $\mathbb{1 1}$ (1975), 31-50. 
[ 8 ] Hamachi, T. and Osikawa, M., Fundamental homomorphism of normalizer group of ergodic transformation, Proc. of Conf. on Ergodic Th. at Oberwolfach, Springer Lecture Notes in Math., 729, 1979, 43-57.

[9] Krieger, W., On ergodic flows and isomorphism of factors, Math. Ann., 223 (1976), $19-70$.

[10] Ornstein, D., On the root problem in ergodic theory, Proc. 6th Berkeley Symp. Math. Statist. Probability, 2 (1972), 347-356.

[11] Ornstein, D., Imbedding Bernoulli shifts in flows, Springer Lecture Notes in Math., 160, 1970, 178-218. 